\title{
Predictor of 90-day functional outcome after mechanical thrombectomy for large vessel occlusion stroke: NIHSS score of 10 or less at 24 hours
}

\author{
Mirja M. Wirtz, MD, ${ }^{1}$ Philipp Hendrix, MD, ${ }^{1,2}$ Oded Goren, MD, ${ }^{1}$ Lisa A. Beckett, RN, ${ }^{3}$ \\ Heather R. Dicristina, RN, ${ }^{3}$ Clemens M. Schirmer, MD, PhD, ${ }^{1,6}$ Shamsher Dalal, MD, EMBA, ${ }^{1,4}$ \\ Gregory Weiner, MD, ${ }^{1}$ Paul M. Foreman, MD, ${ }^{1,5}$ Ramin Zand, MD, MPH, ${ }^{3}$ and \\ Christoph J. Griessenauer, MD ${ }^{1,6}$
}

Departments of ${ }^{1}$ Neurosurgery, ${ }^{3}$ Neurology, and ${ }^{4}$ Radiology, Geisinger, Danville, Pennsylvania; ${ }^{2}$ Department of Neurosurgery, Saarland University Medical Center and Saarland University Faculty of Medicine, Homburg, Germany; ${ }^{5}$ Orlando Health, Neuroscience and Rehabilitation Institute, Orlando, Florida; and ${ }^{6}$ Research Institute of Neurointervention, Paracelsus Medical University, Salzburg, Austria

OBJECTIVE Mechanical thrombectomy is the established treatment for acute ischemic stroke due to large vessel occlusion (LVO). The authors sought to identify early predictors of a favorable outcome in stroke patients treated with mechanical thrombectomy.

METHODS Consecutive patients with ischemic stroke due to LVO who underwent mechanical thrombectomy at a Comprehensive Stroke Center in the US between 2016 and 2018 were retrospectively reviewed. Demographics, stroke and treatment characteristics, as well as functional outcome at 90 days were collected. Clinical predictors of 90 -day functional outcome were assessed and compared to existing indices for prompt neurological improvement. Analyses of area under the receiver operating characteristic curve were performed to estimate the optimal thresholds for absolute 24-hour and delta (change in) National Institutes of Health Stroke Scale (NIHSS) scores for functional outcome prediction.

RESULTS A total of 156 patients (median age 71.5 years) underwent 159 mechanical thrombectomies. The $M_{1}$ segment of the middle cerebral artery was the most frequent site of occlusion (57.2\%). The median NIHSS score before thrombectomy was 18 (IQR 14-24). A postthrombectomy Thrombolysis in Cerebral Infarction score of 2B or 3 was achieved in 147 procedures (92.4\%). The median NIHSS score 24 hours after thrombectomy was 14 (IQR 6-22). Good functional outcome at 90 days (modified Rankin Scale score 0-2) was achieved in 37 thrombectomies (23.9\%). An absolute 24hour NIHSS score $\leq 10$ (OR 25.929, 95\% CI 8.448-79.582, $\mathrm{p}<0.001$ ) and a delta NIHSS score $\geq 8$ between baseline and 24 hours (OR 4.929, 95\% Cl 2.245-10.818, p < 0.001) were associated with good functional outcome at 90 days. The 24-hour NIHSS score cutoff of 10 outperformed existing indices for prompt neurological improvement in the ability to predict 90 -day functional outcome.

CONCLUSIONS An NIHSS score $\leq 10$ at 24 hours after mechanical thrombectomy was independently associated with good functional outcome at 90 days.

https://thejns.org/doi/abs/10.3171/2019.10.JNS191991

KEYWORDS large vessel occlusion; thrombectomy; stroke scale; outcome; ischemic; endovascular; interventional; vascular disorders

$\mathrm{T}$ $\mathrm{HE}$ benefit of mechanical thrombectomy for the treatment of acute ischemic stroke associated with large vessel occlusion (LVO) up to 24 hours from stroke onset is well established. ${ }^{2,8}$ Secondary analyses of the DEFUSE 3 (Endovascular Therapy Following Imag- ing Evaluation for Ischemic Stroke 3) trial showed that this benefit prevailed across a broad range of demographic and stroke characteristics such as patient age, stroke severity, time to randomization, imaging modality, and LVO location in the anterior circulation.? The benefit is

ABBREVIATIONS ASPECTS = Alberta Stroke Program Early CT Score; AUC = area under the receiver operating characteristic curve; CSC = Comprehensive Stroke Center; DEFUSE 3 = Endovascular Therapy Following Imaging Evaluation for Ischemic Stroke 3; DNI = dramatic neurological improvement; ENI = early neurological improvement; IQR = interquartile range; LVO = large vessel occlusion; $\mathrm{mRS}=$ modified Rankin Scale; NIHSS = National Institutes of Health Stroke Scale; RNI = rapid neurological improvement; $\mathrm{TICl}=$ Thrombolysis in Cerebral Infarction; tPA = tissue plasminogen activator.

SUBMITTED July 22, 2019. ACCEPTED October 8, 2019.

INCLUDE WHEN CITING Published online December 20, 2019; DOI: 10.3171/2019.10.JNS191991. 
usually determined in terms of the proportion of patients achieving a modified Rankin Scale (mRS) score of 0-2 (i.e., functional independence) at 90 days. Early predictors of functional recovery could assist in guiding patient and family expectations while also informing decisions regarding the most appropriate rehabilitation programs and disposition. Early neurological recovery within a matter of hours or a few days from stroke intervention has been identified as an important predictor of long-term outcome in thrombolysis ${ }^{4,6,11}$ as well as mechanical thrombectomy cohorts. ${ }^{3}$ Whether this observation remains true outside of well-designed prospective clinical trials is less certain. In this study we sought to assess early clinical predictors of long-term favorable functional outcome at a Comprehensive Stroke Center (CSC) in patients undergoing mechanical thrombectomy for acute ischemic stroke secondary to LVO.

\section{Methods \\ Data Collection}

Consecutive patients with acute ischemic stroke due to LVO who underwent mechanical thrombectomy at a CSC in the US between February 2016 and December 2018 were retrospectively reviewed. IRB approval was obtained. No patients were excluded from the analysis. LVOs in the anterior and posterior circulation were included. Demographics, stroke and thrombectomy characteristics, National Institutes of Health Stroke Scale (NIHSS) scores at presentation and 24 hours after treatment, and functional outcome at 90 days as measured by the $\mathrm{mRS}$ were obtained. Data collection was performed by a medical professional not involved in the procedures or management of the patients (M.M.W.).

\section{Mechanical Thrombectomy Procedure}

Patients were evaluated and treated according to current American Heart Association and American Stroke Association guidelines, including evaluation by a multidisciplinary stroke team and consideration for intravenous thrombolysis. ${ }^{9}$ Mechanical thrombectomy was performed under conscious sedation or general anesthesia at the discretion of the treating neurointerventionalist and anesthesiologist. Femoral access using an 8-Fr groin sheath was obtained except for selected cases in which radial or brachial approaches were deemed more appropriate. The decision to use a balloon guide catheter was made at the discretion of the neurointerventionalist. Thrombectomy was performed using state-of-the-art devices and included either large-bore aspiration catheters or stent retrievers, or a combination of both. The access sites were closed using closure devices or manual compression.

\section{Comparison to Existing Indices of Prompt Neurological Improvement}

Accuracy of rapid neurological improvement (RNI, defined as a reduction of $\geq 8$ on the NIHSS or an NIHSS score $<2$ at 24 hours after thrombectomy), ${ }^{3}$ dramatic neurological improvement (DNI, defined as a reduction of $\geq$ 10 on the NIHSS or an NIHSS score $<4$ at 24 hours), ${ }^{1,6}$ and early neurological improvement (ENI, defined as an improvement in NIHSS score $>4$ at 24 hours), ${ }^{10}$ were assessed and compared to predictors in the current cohort.

\section{Statistical Analyses}

Continuous variables are presented as means and interquartile ranges (IQRs), and categorical variables are presented as frequencies and percentages. Analyses were performed using the chi-square test, Fisher's exact test, Mann-Whitney U-test, and univariable binary logistic regression as appropriate. Multivariable binary logistic regression analysis was performed by integrating variables that found an association with 90-day functional outcome in univariable analysis $(p<0.15)$. Stepwise backward elimination was performed, and $\mathrm{p}$ values $<0.05$ were considered statistically significant. Collinearity diagnostics were performed, assessing correlation coefficients and variance inflation factors.

Analyses of area under the receiver operating characteristic curve (AUC) were performed to estimate the optimal threshold for absolute 24-hour and delta (change in) NIHSS 90-day functional outcome prediction. Optimal thresholds were calculated using 1000 bootstrap replications and maximizing Youden's J for baseline NIHSS score at presentation, NIHSS score 24 hours after mechanical thrombectomy, delta NIHSS score, and combined models (delta NIHSS score and/or NIHSS score 24 hours after mechanical thrombectomy).

Next, optimal thresholds were compared to identify the strongest predictor for 90-day functional outcome of the present study. Additionally, optimal thresholds were compared to other previously established predictors of functional outcome (RNI, DNI, and ENI). Comparison of AUCs was performed using DeLong tests. Statistical analyses and the creation of figures and charts were performed using IBM SPSS (version 22, IBM Corp.), MedCalc (version 19.0.3), Microsoft Excel, and Adobe Photoshop CS5.

\section{Results \\ Baseline Characteristics}

During the study period, 159 mechanical thrombectomies for acute ischemic stroke due to LVO were performed in 156 patients (median age 71.5 years, IQR 61-82 years; $52 \%$ women). Hypertension (76.7\%), hyper- or dyslipidemia (73\%), and atrial fibrillation $(47.8 \%)$ were the most common risk factors. Intravenous tissue plasminogen activator (tPA) was administered in $40.3 \%$ of cases. The majority of strokes were due to either cardioembolism $(49.1 \%)$ or large-artery atherosclerosis (23.9\%). A premorbid mRS score of 0-2 was recorded in 138 procedures (90.2\%). The median NIHSS score before thrombectomy was 18 (IQR 14-24; Table 1).

\section{Mechanical Thrombectomy Procedure}

The $\mathrm{M}_{1}$ segment of the middle cerebral artery and the internal carotid artery were most frequently occluded (57.2\% and $23.3 \%$, respectively). Tandem occlusions were encountered in 21 procedures (13.2\%). A combination of aspiration catheter and stent retriever was most commonly used (86.2\%). A postthrombectomy Thrombolysis in Cerebral Infarction (TICI) grade of $2 \mathrm{~B}$ or 3 was achieved in 
TABLE 1. Baseline characteristics

\begin{tabular}{|c|c|}
\hline Parameter & Value \\
\hline No. of patients & 156 \\
\hline Females, $\mathrm{n}(\%)$ & $81(51.9)$ \\
\hline Median age (IQR), yrs & $71.5(61-82)$ \\
\hline No. of procedures, $\mathrm{n}(\%)$ & 159 \\
\hline Hypertension & $122(76.7)$ \\
\hline Hyper- or dyslipidemia & $116(73)$ \\
\hline Atrial fibrillation & $76(47.8)$ \\
\hline Chronic kidney disease & $44(27.7)$ \\
\hline Diabetes mellitus & $42(26.4)$ \\
\hline Coronary artery disease & $40(25.2)$ \\
\hline Ischemic stroke & $22(13.8)$ \\
\hline Transient ischemic attack & $10(6.3)$ \\
\hline \multicolumn{2}{|l|}{ Smoker, n (\%) } \\
\hline Never & $68(42.8)$ \\
\hline Former & $50(31.4)$ \\
\hline Current & $41(25.8)$ \\
\hline \multicolumn{2}{|l|}{ Presentation, n (\%) } \\
\hline Transferred & $90(56.6)$ \\
\hline Presented to stroke center & $69(43.4)$ \\
\hline Intravenous tPA, n (\%) & $64(40.3)$ \\
\hline \multicolumn{2}{|l|}{ Etiology, n (\%) } \\
\hline Cardioembolic & $78(49.1)$ \\
\hline Large-artery atherosclerosis & $38(23.9)$ \\
\hline Unknown & $25(15.7)$ \\
\hline Other & $18(11.3)$ \\
\hline \multicolumn{2}{|l|}{ Premorbid mRS score, $\mathrm{n}(\%)^{*}$} \\
\hline $0-2$ & $138(90.2)$ \\
\hline $3-5$ & $15(9.8)$ \\
\hline \multicolumn{2}{|l|}{ ASPECTS, n (\%)† } \\
\hline$<6$ & $8(5.2)$ \\
\hline$\geq 6$ & $145(94.8)$ \\
\hline \multicolumn{2}{|l|}{ NIHSS score, $\mathrm{n}(\%) \ddagger$} \\
\hline Median (IQR) & $18(14-24)$ \\
\hline$<6$ & $4(2.5)$ \\
\hline $6-15$ & $45(28.5)$ \\
\hline $16-20$ & $46(29.1)$ \\
\hline$\geq 21$ & $63(39.9)$ \\
\hline \multicolumn{2}{|l|}{ LVO location, n (\%) } \\
\hline$M_{1}$ & $91(57.2)$ \\
\hline Internal carotid artery & $37(23.3)$ \\
\hline $\mathrm{M}_{2}$ & $16(10.1)$ \\
\hline Basilar artery & $13(8.2)$ \\
\hline Vertebral artery & $1(0.6)$ \\
\hline $\mathrm{A}_{3}$ & $1(0.6)$ \\
\hline \multicolumn{2}{|l|}{ Laterality, n (\%) } \\
\hline $\mathrm{Rt}$ & $76(47.8)$ \\
\hline $\mathrm{Lt}$ & $69(43.4)$ \\
\hline Tandem occlusion & $21(13.2)$ \\
\hline
\end{tabular}

» CONTINUED FROM PREVIOUS COLUMN

\section{TABLE 1. Baseline characteristics}

\begin{tabular}{|c|c|}
\hline Parameter & Value \\
\hline \multicolumn{2}{|l|}{ Mechanical thrombectomy, $\mathrm{n}(\%)$} \\
\hline Aspiration + stent retriever & $137(86.2)$ \\
\hline Aspiration only & $13(8.2)$ \\
\hline Stent retriever only & $9(5.6)$ \\
\hline \multicolumn{2}{|l|}{$\mathrm{TICl}$ grade, $\mathrm{n}(\%)$} \\
\hline 3 & $88(55.3)$ \\
\hline $2 \mathrm{~B}$ & $59(37.1)$ \\
\hline $2 \mathrm{~A}$ & $6(3.8)$ \\
\hline 1 & $4(2.5)$ \\
\hline 0 & $2(1.3)$ \\
\hline \multicolumn{2}{|l|}{ Carotid/vertebral artery occlusion, $\mathrm{n}(\%)$} \\
\hline Angioplasty only & $12(7.5)$ \\
\hline Stenting only & $6(3.8)$ \\
\hline Angioplasty + stenting & $4(2.5)$ \\
\hline Acute intracranial stenting, $\mathrm{n}(\%)$ & $1(0.6)$ \\
\hline $\begin{array}{l}\text { Median interval btwn last known well time \& presenta- } \\
\text { tion to CSC (IQR), mins§ }\end{array}$ & $247(122-505)$ \\
\hline $\begin{array}{l}\text { Median interval btwn time of presentation to CSC \& } \\
\text { time of intervention (IQR), mins }\end{array}$ & $53(25-103)$ \\
\hline $\begin{array}{l}\text { Median interval btwn time of intervention \& revascu- } \\
\text { larization (IQR), mins }{ }^{* *}\end{array}$ & $37(23-70)$ \\
\hline \multicolumn{2}{|l|}{ NIHSS score at $24 \mathrm{hrs}, \mathrm{n}(\%)+\dagger$} \\
\hline Median (IQR) & $14(6-22)$ \\
\hline$<6$ & $35(22.2)$ \\
\hline $6-15$ & $49(31.0)$ \\
\hline $16-20$ & $30(19.0)$ \\
\hline$\geq 21$ & $44(27.8)$ \\
\hline $\begin{array}{l}\text { Median no. of nights in hospital after intervention date } \\
\text { (IQR) }\end{array}$ & $6(4-12)$ \\
\hline \multicolumn{2}{|l|}{ Disposition on discharge, $\mathrm{n}(\%)$} \\
\hline Rehabilitation & $63(39.6)$ \\
\hline Dead & $34(21.4)$ \\
\hline Home & $28(17.6)$ \\
\hline Skilled nursing facility & $21(13.2)$ \\
\hline Long-term acute care hospital & $9(5.7)$ \\
\hline Hospice & $4(2.5)$ \\
\hline \multicolumn{2}{|l|}{ 90-day mRS score, n (\%)㧊 } \\
\hline $0-2$ & $37(23.9)$ \\
\hline $3-5$ & $69(44.5)$ \\
\hline 6 & $49(31.6)$ \\
\hline
\end{tabular}

* Data missing for 6 procedures.

$\dagger$ Data missing for 6 procedures.

$\ddagger$ Data not available for 1 procedure.

$\S$ Data not available for 13 procedures.

TI Data not available for 10 procedures.

** Data not available for 2 procedures.

†† Data not available for 1 procedure.

拉 Data not available for 4 procedures. 
TABLE 2. Factors associated with functional outcome

\begin{tabular}{|c|c|c|c|}
\hline \multirow[b]{2}{*}{ Variable } & \multicolumn{2}{|c|}{ 90-Day mRS Score $(n=155)^{*}$} & \multirow[b]{2}{*}{ OR $(95 \% \mathrm{Cl}), \mathrm{p}$ Value } \\
\hline & $0-2(n=37)$ & $3-6(n=118)$ & \\
\hline Female sex & $21(56.8 \%)$ & $57(48.3 \%)$ & 1.405 (0.668-2.955), 0.371 \\
\hline Median age (IQR) & $64(55-79)$ & $73(65-82)$ & $p=0.02$ \\
\hline Hypertension & $21(56.8 \%)$ & $98(83.1 \%)$ & 0.268 (0.119-0.601), 0.001 \\
\hline Diabetes mellitus & $6(16.2 \%)$ & $36(30.5 \%)$ & 0.441 (0.169-1.149), 0.088 \\
\hline Hyper- or dyslipidemia & $24(64.9 \%)$ & $89(75.4 \%)$ & 0.602 (0.272-1.331), 0.207 \\
\hline Coronary artery disease & $6(16.2 \%)$ & $33(28.0 \%)$ & 0.499 (0.190-1.305), 0.151 \\
\hline Atrial fibrillation & $13(35.1 \%)$ & $61(51.7 \%)$ & 0.506 (0.235-1.088), 0.078 \\
\hline Chronic kidney disease & $13(35.1 \%)$ & $31(26.3 \%)$ & $1.520(0.690-3.349), 0.297$ \\
\hline Ischemic stroke & $2(5.4 \%)$ & $19(16.1 \%)$ & 0.298 (0.066-1.344), 0.097 \\
\hline Transient ischemic attack & $0(0.0 \%)$ & $10(8.5 \%)$ & $p=0.119$ \\
\hline Smoking (current/former) & $19(51.4 \%)$ & $70(59.3 \%)$ & $0.724(0.345-1.520), 0.392$ \\
\hline Median baseline NIHSS score (IQR) & $14(10-19)$ & $20(16-26)$ & $p<0.001$ \\
\hline Anterior circulation location of LVO & $36(97.3 \%)$ & $105(89 \%)$ & 4.457 (0.563-35.282), 0.190 \\
\hline ASPECTS $\geq 6$ & $37(100.0 \%)$ & $104(92.9 \%) \dagger$ & $p=0.201$ \\
\hline Tandem occlusion & $6(16.2 \%)$ & $14(11.9 \%)$ & $1.438(0.510-4.056), 0.574$ \\
\hline Last known well to revascularization $<6 \mathrm{hrs}$ & $20(58.8 \%) \ddagger$ & $52(48.6 \%) \S$ & $1.511(0.692-3.300), 0.299$ \\
\hline Transfer to CSC & 17 (48.6\%)ף & $69(61.6 \%)^{* *}$ & 0.589 (0.274-1.264), 0.172 \\
\hline Revascularization ( $\mathrm{TICl}$ grade 2B \& 3) & $37(100.0 \%)$ & $106(89.8 \%)$ & $p=0.071$ \\
\hline Intravenous tPA & $20(54.1 \%)$ & $44(37.3 \%)$ & 1.979 (0.938-4.174), 0.071 \\
\hline \multicolumn{4}{|l|}{ NIHSS score at $24 \mathrm{hrs}$} \\
\hline Median (IQR) & $4(2-8)$ & $17(10-23)$ & $p<0.001$ \\
\hline$\leq 10$ & $33(89.2 \%)$ & $28(24.1 \%) \dagger \dagger$ & $25.929(8.448-79.582),<0.001$ \\
\hline \multicolumn{4}{|l|}{ Delta NIHSS score at $24 \mathrm{hrs}$} \\
\hline Median (IQR) & $9(2-13)$ & $3(-1$ to 8$)$ & $p<0.001$ \\
\hline$\geq 8$ & $23(62.2 \%)$ & $29(25.0 \%) \dagger \dagger$ & $4.929(2.245-10.818),<0.001$ \\
\hline $\begin{array}{l}\text { * Data not available for } 4 \text { procedures. } \\
\text { † Data missing for } 6 \text { procedures. } \\
\text { † Data missing for } 3 \text { procedures. } \\
\text { § Data missing for } 11 \text { procedures. } \\
\text { I Data missing for } 2 \text { procedures. } \\
\text { ** Data missing for } 6 \text { procedures. } \\
\dagger † \text { Data missing for } 2 \text { procedures. }\end{array}$ & & & \\
\hline
\end{tabular}

147 procedures $(92.5 \%)$. Median times from presentation to arterial puncture and arterial puncture to revascularization were 53 and 37 minutes, respectively (Table 1).

\section{Early Neurological Recovery and Functional Outcome}

The median NIHSS score 24 hours after thrombectomy was 14 (IQR 6-22). Good functional outcome at 90 days ( $\mathrm{mRS}$ score $0-2$ ) was achieved in 37 thrombectomies (23.9\%, Table 1). History of hypertension (OR $0.268,95 \%$ CI $0.119-0.601, p=0.001)$, baseline NIHSS score $(\mathrm{p}<0.001)$, NIHSS score $\leq 10$ at 24 hours (OR $25.929,95 \%$ CI $8.448-79.582, \mathrm{p}<0.001)$, and improvement in NIHSS score $\geq 8$ at 24 hours (OR 4.929, 95\% CI $2.245-10.818, \mathrm{p}<0.001)$ were associated with functional independence (mRS score 0-2) at 90 days (Table 2). In multivariable analysis, history of hypertension (OR 0.225 , $95 \%$ CI $0.077-0.659, p=0.006)$ and an NIHSS score $\leq 10$ at 24 hours (OR 28.296, 95\% CI 8.732-91.693, p < 0.001) remained significantly associated with functional inde- pendence (mRS score 0-2) at 90 days. The results did not significantly change when the analysis was performed per patient instead of per thrombectomy (Table 3).

\section{Comparison to Existing Indices of Prompt Neurological Improvement}

In the present cohort, the NIHSS score $\leq 10$ at 24 hours had an AUC of 0.825 (95\% CI $0.756-0.882$, p < 0.0001). The Youden index $\mathrm{J}$ was 0.65 with $89.2 \%$ sensitivity and $75.9 \%$ specificity. The improvement in NIHSS score of $\geq$ 8 at 24 hours had an AUC of 0.686 (95\% CI 0.606-0.758, $\mathrm{p}<0.0001)$. The Youden index J was 0.37 with $62.2 \%$ sensitivity and $75 \%$ specificity.

Calculated scores for RNI (AUC 0.713. 95\% CI 0.6340.783, p < 0.0001), DNI (AUC 0.669, 95\% CI 0.619-0.77, p $<0.0001$ ), and ENI (AUC 0.617, 95\% CI 0.535-0.695, p < 0.0106 ) were all found to be inferior to NIHSS score $\leq 10$ at 24 hours in the present cohort (DeLong tests for RNI [p $=0.0108]$, DNI [ $p=0.0060]$, and ENI [p < 0.0001]; Fig. 1). 
TABLE 3. Multivariable analysis of factors associated with 90 -day functional outcome (per thrombectomies and per patients)

\begin{tabular}{|c|c|c|c|}
\hline Predictors & $\beta$ Coefficient & OR $(95 \% \mathrm{Cl})$ & p Value \\
\hline \multicolumn{4}{|l|}{ Analysis per 155 thrombectomies } \\
\hline Hypertension & -1.49 & $0.23(0.08-0.7)$ & 0.006 \\
\hline NIHSS score $\leq 10$ at $24 \mathrm{hrs}$ & 3.34 & $28.3(8.73-91.69)$ & $<0.001$ \\
\hline \multicolumn{4}{|l|}{ Analysis per 152 patients } \\
\hline Hypertension & -1.56 & $0.21(0.07-0.62)$ & 0.005 \\
\hline NIHSS score $\leq 10$ at 24 hrs & 3.12 & $22.53(7.83-64.76)$ & $<0.001$ \\
\hline
\end{tabular}

\section{Discussion}

Prompt neurological improvement is the hallmark of success for mechanical thrombectomy in the treatment of acute ischemic stroke due to LVO. Significant improvements in signs and symptoms in thrombectomy patients while still on the angiography table are most memorable among members of the thrombectomy team. In the present study, a history of hypertension, baseline NIHSS score, NIHSS score $\leq 10$ at 24 hours, and improvement in NIHSS score $\geq 8$ at 24 hours were associated with functional independence (mRS score 0-2) at 90 days. In the multivariable analysis, a history of hypertension and an NIHSS score $\leq 10$ at 24 hours after thrombectomy remained significant. This cohort represents a real-world sample of patients undergoing mechanical thrombectomy, including patients with poor functional status prior to thrombectomy (mRS score 3-5), low Alberta Stroke Program Early CT Score (ASPECTS; <6), patients with extended times from "Last Known Well," and LVO in the posterior circulation. As expected, this results in a drop in the proportion of patients achieving functional independence. Interestingly, the NIHSS score at 24 hours was the strongest predictor of long-term functional outcome and outperformed all assessed and previously proposed measures ${ }^{1,3,6,10}$ of prompt neurological improvement. This novel finding lends itself as not only an appropriate but also simple and practical predictor applicable to clinical practice. A cutoff value of 10 may also be preferred to a change in the NIHSS score from before to after thrombectomy, as the latter is heavily influenced by the pretreatment score. For example, a patient presenting with an NIHSS score of 10 who improves to an NIHSS score of 0 after 24 hours has the same 10-point decrease as a patient improving from an NIHSS score of 40 to an NIHSS score of 30 . Yet, the former patient is very likely to be independent at 90 days, whereas the latter may still not achieve functional independence, despite an identical 10-point decrease in NIHSS score.

\section{Comparison to Existing Indices of Prompt Neurological Improvement}

Soize et al. analyzed 246 large-vessel stroke patients treated with thrombectomy and identified the ENI as the most important predictor of 90-day functional outcome. ${ }^{10}$ Despite the larger sample size, the study had a number of limitations. Patients were treated between 2010 and 2017. Given the rapid device development in the neurovascular space, it is unlikely that all patients were treated with state-of-the-art devices such as modern aspiration cath- eters. As previously noted, a delta NIHSS score does not reflect the baseline NIHSS score nor the 24-hour NIHSS score. The latter has shown to be the most important predictor of functional outcome in the present study. Likewise, higher baseline NIHSS score may allow for greater improvement after thrombectomy. Next, the degree of revascularization was significantly different in that study, a bias not present in the current cohort where there was no significant association of revascularization and functional outcome. Lastly, the ENI model for 24 hours listed an OR of 391.32 with a $95 \%$ CI of 44.43-3448.35, significantly wider than in the current study. In our cohort, the ENI had the least ability to distinguish patients achieving favorable outcomes at 90 days.

A recent analysis of the DEFUSE 3 trial showed that the RNI correlated with functional outcome, mortality, infarct volume and growth, and hospital length of stay. ${ }^{3}$ Unfortunately, the data were from a very select group of thrombectomy patients 6-16 hours from Last Known Well. As such, the applicability of the findings in clinical practice are limited. Shortcomings of a delta NIHSS score

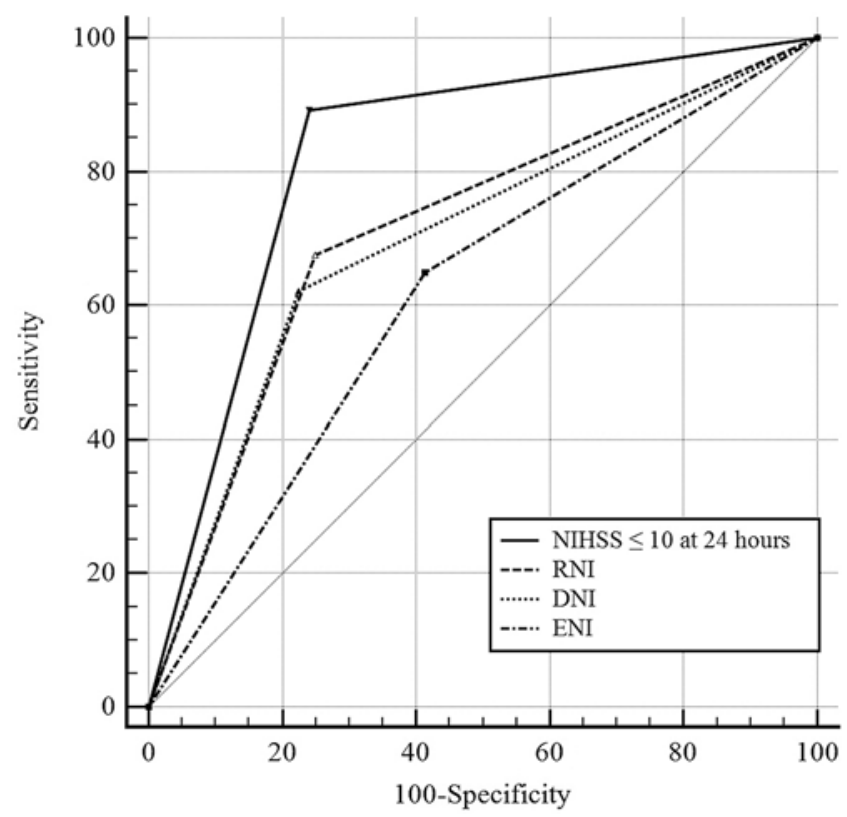

FIG. 1. Receiver operating characteristic curves for NIHSS score $\leq 10$ at 24 hours and for RNI, DNI, and ENI for prediction of functional outcome (mRS score $0-2)$ at 90 days. AUC for NIHSS score $\leq 10$ at 24 hours $=$ 0.825 , for $\mathrm{RNI}=0.713$, for $\mathrm{DNI}=0.669$, and for $\mathrm{ENI}=0.617$. 
improvement have been discussed above. The authors also defined RNI as an absolute NIHSS score of $<2$ at 24 hours. Only a minority of thrombectomies achieve an NIHSS score $<2$ at 24 hours. In our cohort, only 8 thrombectomies (5\%) had an NIHSS score of 0 or 1 at 24 hours. As such, this cutoff is not practical as it may suggest the vast majority of thrombectomy patients may have a poor long-term outcome. However, the RNI was the closest in terms of AUC to the $\leq 10$ NIHSS score cutoff at 24 hours.

The DNI was developed from patients enrolled in the sonothrombolysis trial. ${ }^{1}$ It also showed discriminatory abilities in patients treated with intravenous tPA. ${ }^{6}$ In our cohort, the DNI performed slightly worse than the RNI. Whether prompt neurological improvement definitions from sonothrombolysis or intravenous thrombolysis studies apply to mechanical thrombectomy, is certainly questionable. Mechanical thrombectomy and intravenous or sonothrombolysis may follow different neurological recovery kinetics. ${ }^{10}$ Successful mechanical thrombectomy establishes reperfusion rapidly while intravenous tPA most commonly fails to recanalize proximal LVO due to the longer and more robust clots. ${ }^{5}$ Those studies also included non-LVO strokes that are often associated with lower baseline NIHSS score. Hence, there is less potential for a substantial decrease in NIHSS score. Consistent with those findings, prompt neurological improvement has shown to be more accurate as the baseline NIHSS score increases..$^{10}$ Likewise, neurological improvement of NIHSS score $\geq$ 4 was unable to predict good functional outcome in the large-vessel stroke subset in intravenous tPA and placebo groups. ${ }^{4}$

\section{Study Strengths and Limitations}

In contrast to aforementioned published definitions of prompt neurological improvement, the current study fulfills three critical criteria of valuable scoring systems. First, it is simple as an NIHSS cutoff score of 10 is easy to remember and apply. Second, it was applied in a cohort of patients undergoing mechanical thrombectomy as they are encountered in the real world without any exclusions. Lastly, it only included patients treated in the new era of mechanical thrombectomy where state-of-the-art thrombectomy devices were used.

Nevertheless, the study has limitations. As a retrospective study, the quality of the data may be compromised by missing values, even though NIHSS and mRS scores were recorded in a prospective fashion as required per CSC guidelines. The group of LVO strokes was heterogeneous, which may be seen as a strength, but also a disadvantage. The previously described indices of prompt neurological improvement (RNI, DNI, and ENI) were externally validated in the present study. No prior study had assessed all those indices in the same cohort. Using the same data set, the NIHSS score of $\leq 10$ at 24 hours was developed, but has not yet been externally validated. Therefore, the findings of this study warrant validation in independent, external cohorts.

\section{Conclusions}

An NIHSS score $\leq 10$ at 24 hours after thrombectomy was independently associated with functional independence (mRS score 0-2) at 90 days. In the present study, this straightforward cutoff of NIHSS score at 24 hours outperformed previously published prompt neurological recovery scoring systems and may be useful in discussions with patients, their family members, and members of the care team to prognosticate functional outcome shortly after procedure completion.

\section{References}

1. Alexandrov AV, Molina CA, Grotta JC, Garami Z, Ford SR, Alvarez-Sabin J, et al: Ultrasound-enhanced systemic thrombolysis for acute ischemic stroke. N Engl J Med 351:21702178, 2004

2. Goyal M, Menon BK, van Zwam WH, Dippel DWJ, Mitchell PJ, Demchuk AM, et al: Endovascular thrombectomy after large-vessel ischaemic stroke: a meta-analysis of individual patient data from five randomised trials. Lancet 387:17231731,2016

3. Heit JJ, Mlynash M, Kemp SM, Lansberg MG, Christensen S, Marks MP, et al: rapid neurologic improvement predicts favorable outcome 90 days after thrombectomy in the DEFUSE 3 study. Stroke 50:1172-1177, 2019

4. Hemmen TM, Ernstrom K, Raman R: Two-hour improvement of patients in the National Institute of Neurological Disorders and Stroke trials and prediction of final outcome. Stroke 42:3163-3167, 2011

5. Kamalian S, Morais LT, Pomerantz SR, Aceves M, Sit SP, Bose A, et al: Clot length distribution and predictors in anterior circulation stroke: implications for intra-arterial therapy. Stroke 44:3553-3556, 2013

6. Kharitonova T, Mikulik R, Roine RO, Soinne L, Ahmed $\mathrm{N}$, Wahlgren N: Association of early National Institutes of Health Stroke Scale improvement with vessel recanalization and functional outcome after intravenous thrombolysis in ischemic stroke. Stroke 42:1638-1643, 2011

7. Lansberg MG, Mlynash M, Hamilton S, Yeatts SD, Christensen S, Kemp S, et al: Association of thrombectomy with stroke outcomes among patient subgroups: secondary analyses of the DEFUSE 3 randomized clinical trial. JAMA Neurol 76:447-453, 2019

8. Nogueira RG, Jadhav AP, Haussen DC, Bonafe A, Budzik RF, Bhuva P, et al: Thrombectomy 6 to 24 hours after stroke with a mismatch between deficit and infarct. $\mathbf{N}$ Engl J Med 378:11-21, 2018

9. Powers WJ, Rabinstein AA, Ackerson T, Adeoye OM, Bambakidis NC, Becker K, et al: 2018 Guidelines for the early management of patients with acute ischemic stroke: a guideline for healthcare professionals from the American Heart Association/American Stroke Association. Stroke 49:e46e110, 2018

10. Soize S, Fabre G, Gawlitza M, Serre I, Bakchine S, Manceau $\mathrm{PF}$, et al: Can early neurological improvement after mechanical thrombectomy be used as a surrogate for final stroke outcome? J Neurointerv Surg 11:450-454, 2019

11. Yeo LLL, Paliwal P, Teoh HL, Seet RC, Chan BPL, Wakerley $\mathrm{B}$, et al: Early and continuous neurologic improvements after intravenous thrombolysis are strong predictors of favorable long-term outcomes in acute ischemic stroke. J Stroke Cerebrovasc Dis 22:e590-e596, 2013

\section{Disclosures}

The authors report no conflict of interest concerning the materials or methods used in this study or the findings specified in this paper. 


\section{Author Contributions}

Conception and design: Griessenauer. Acquisition of data: Griessenauer, Wirtz, Beckett, Dicristina. Analysis and interpretation of data: Griessenauer, Wirtz, Hendrix. Drafting the article: Griessenauer, Wirtz. Critically revising the article: Griessenauer, Wirtz, Hendrix, Goren, Schirmer, Dalal, Weiner, Foreman, Zand. Reviewed submitted version of manuscript: Griessenauer, Hendrix, Goren, Beckett, Dicristina, Schirmer, Dalal, Weiner, Foreman, Zand. Approved the final version of the manuscript on behalf of all authors: Griessenauer. Statistical analysis: Griessenauer, Wirtz, Hendrix. Administrative/technical/ material support: Griessenauer. Study supervision: Griessenauer.

\section{Correspondence}

Christoph J. Griessenauer: Geisinger, Danville, PA. christoph. griessenauer@gmail.com. 